\title{
New Cross-correlation Results for Multi-rate CDMA
}

\author{
Eric Hamelin, Leslie A. Rusch and Paul Fortier \\ (hamelin@gel.ulaval.ca, fortier@gel.ulaval.ca, rusch@gel.ulaval.ca) \\ Department of Electrical and Computer Engineering \\ Université Laval, Québec, Canada G1K 7P4 \\ (418) 656-2906, (418) 656-3159 fax
}

\begin{abstract}
Support of multiple data services can be accomplished via code division multiple access (CDMA) with a constant chip rate, but variable data rates. We present exact equations for the cross-correlation of codes in a multiple-data rate system, and thus equations for the multiple access interference (MAI) and bit error rate (BER). Previous analysis has found the BER using the mean of the MAI assuming codes are truly random sequences. We show that Gold and Kasami sequences have a cross-correlation with a distribution closely approximated by a Gaussian one, and present confidence intervals to quantify the performance under the random code assumption.
\end{abstract}

\section{Introduction}

New video and multimedia applications require more bandwidth than classical applications with whom they must coexist. One popular approach to share bandwidth is via the use of code division multiple access (CDMA). We examine multirate CDMA systems with fixed chip rate for all users, but varying sequence length according to the data rate required.

Seminal work on single rate systems by Pursley and Sarwate examined the cross-correlation properties of pseudorandom codes for CDMA [1-2]. They considered the popular binary shift-register sequences (m-sequences, Gold codes and Kasami sequences) and characterized their performance via what they defined as an average interference parameter. As bounds on this parameter were too loose to be of value, the mean (calculated by modeling the pseudo-random codes as truly random) was used to evaluate performance.

Ottosson and Svensson [3] extended single rate results to multi-rate CDMA systems with constant chip rates using the random sequence assumption. As they were only interested in the mean value of the average interference parameter, they bypassed calculation of the exact value of the parameter as a function of actual code values.

In this work we determine an exact equation for the average interference parameter for the multi-rate CDMA system using actual sequences. For single rate systems the average interference parameter involves correlation over two partially interfering bits, while the multi-rate system can have many overlapping interference bits leading to much more complicated expressions for the average interference parameter.

Extensive calculation of the exact interference parameter among actual code sequences led us to observe a Gaussian distribution. Consider for example a single user system with length 127 codes, and 127 distinct codes. There are $127 \times 126$ distinct average interference parameters depending upon the particular pair of codes considered. If we consider the choice of active codes from the set of 127 deterministic codes to be ran- dom, the interference parameter is observed to have a Gaussian distribution, for both single-rate or multi-rate systems. Examination of several code families shows that the mean of the Gaussian distribution coincides with the mean under the random code assumption. By assuming a Gaussian distribution for the code, we are able to quantify (via confidence intervals) the value of the random code assumption.

\section{Multi-rate DS-CDMA System Model}

We use direct sequence (DS) CDMA with binary phase shift keying (BPSK). Each user transmits independently of other users, and the system can support $p$ different data rates (subsystems). The transmitted signal of user $k$ in subsystem $i$ is

$$
s_{i k}(t)=\sqrt{2 P_{i}} b_{i k}(t) a_{i k}(t) \cos \omega_{c} t+\theta_{i k} \text { ! }
$$

where $\omega_{c}$ is the carrier frequency, $\theta_{i k}$ is the initial phase offset (modeled as a sequence of independent random variables uniformly distributed over $[0,2 \pi]), b_{i k}(t)$ is the data bit of user $k$ of subsystem $i$ at time $t$ (modeled as a set of independent equiprobable random variables taking values in $\{-1,1\}), a_{i k}(t)$ is the spreading code of period $N_{i}$ of user $k$ of subsystem $i$ (taking values in $\{-1,1\})$, and $P_{i}=E_{b} / T_{i}$ is the average power corresponding to data rate $R_{i}=1 / T_{i}$, where $T_{i}$ is the bit interval on subsystem $i$. We assume the spreading code $a_{i k}(t)$ consists of rectangular pulse shapes with chip pulse duration $T_{c}=T_{i} / N_{i}$. The signal's average power is different for each data rate, with constant energy per bit [3], i.e., $E_{b}=P_{i} T_{i}=P_{j} T_{j}$. The received signal is at any network node is given by

$$
r(t)=w(t)+\sum_{i=1}^{p} \sum_{k=1}^{K_{i}} s_{i k}\left(t-\tau_{i k}\right)
$$

where $w(t)$ is the channel noise modeled as zero mean additive white Gaussian noise (AWGN), $K_{i}$ is the number of simultaneous users with data rate $i$, and $\tau_{i k}$ is the relative delay of user $k$ of subsystem $i$ (modeled as a set of independent random variables uniformly distributed over $\left[0, T_{i}\right]$ ).

While sub-optimal for this channel, we nonetheless adopt the matched filter detector due to its simplicity and widespread use. The receiver matched to user $l$ in subsystem $j$ has output

$$
Z_{j l}={ }^{\boldsymbol{T}_{j}} r(t) a_{j l}(t) \cos \left(\omega_{c} t\right) d t
$$

where $T_{j}$ is the desired signal's bit duration and $a_{j l}(t)$ is its spreading code sequence of the desired signal. A sequence has degree $n$ for period $N=2^{n}-1$. Given two sequences of different lengths $L=2^{\left(n_{y}-n_{x}\right)}$ is the ratio between the sequence lengths. 


\section{Analysis}

The matched filter output $Z_{j l}$ reduces to noise plus

$$
{ }^{\boldsymbol{-}}{ }_{T_{j}} \sum_{i=1}^{p} \sum_{k=1}^{K_{i}} \sqrt{\frac{P_{i}}{2}} b_{i k}\left(t-\tau_{i k}\right) a_{i k}\left(t-\tau_{i k}\right) a_{j l}(t) \cos \left(\varphi_{i k}\right) d t
$$

where the relative phase differences and delays are $\varphi_{i k}=\theta_{i k}-\omega_{c} \tau_{i k}$. The desired signal we have taken to be user $l$ on subsystem $j$, therefore the term $i k=j l$ corresponds to the desired user. Furthermore, we assume coherent detection and ideal timing synchronization between the desired user and the receiver, i.e., $\theta_{j l}=0=\tau_{j l}$ hence $Z_{j l}$ is

$$
\begin{aligned}
& \text { - }_{j} \sqrt{\frac{P_{i}}{2}} b_{j l}(t) a_{j l}^{2}(t) d t+\text { noise } \\
& +\sum_{\substack{i=1 \\
(i k \neq j l)}}^{T} \sum_{k=1}^{K_{i}} \sqrt{\frac{P_{i}}{2}} b_{i k}\left(t-\tau_{i k}\right) a_{i k}\left(t-\tau_{i k}\right) a_{j l}(t) \cos \left(\varphi_{i k}\right) d t
\end{aligned}
$$

where the first integral is the desired signal, the second term is AWGN and the third term is the multiple access interference (MAI). The signal portion can be written as

$$
S_{j l}=\boldsymbol{\sigma}_{j} \sqrt{\frac{P_{j}}{2}} b_{j l}(t) a_{j l}^{2}(t) d t=b_{j l}{ }^{(0)} T_{j} \sqrt{\frac{P_{j}}{2}}
$$

where $b_{j l}^{(0)}$ is the desired user's data bit. The MAI

$$
I_{j l}=\sum_{0}^{T_{i}} \sum_{k=1}^{K_{i}} \sqrt{\frac{P_{i}}{2}} b_{i k}\left(t-\tau_{i k}\right) a_{i k}\left(t-\tau_{i k}\right) a_{j l}(t) \cos \left(\varphi_{i k}\right) d t(7)
$$

is the sum of all non-desired users transmitting asynchronously at various rates in the shared medium with their associated power levels, delays and signature sequences.

In order to determine the bit error rate performance of any CDMA system we must evaluate the statistics of the MAI. We assume that the interference has a Gaussian distribution (justified under certain conditions by the central limit theorem), it suffices to determine the first and second moments of the MAI.

For the case of single-rate CDMA, exact expressions for the variance of the MAI have been found in [1], however due to the difficulty in interpreting this expression for specific codes, research has focused on approximations, modeling codes as truly random sequences of \pm 1 [1]. Under this assumption the variance is averaged over the distribution of the random codes, effectively eliminating the characteristics of the particular type of code in use (e.g., m-sequence, Gold, Kasami, etc.). The only results for multi-rate CDMA with constant chip rate have employed the random code assumption [1]. In the following section we determine the exact expression for the variance of the MAI as a function of the actual codes in use.

\subsection{Multiple Access Interference (MAI)}

Assuming equiprobable bipolar data, the MAI has zero mean and the variance $\operatorname{var}\left[I_{j l}\right]$ is given by $\sum_{\substack{i=1 \\(i \neq j l)}}^{p} \sum_{k=1}^{K_{i}} \frac{1}{T_{j}^{2}} \frac{P_{i}}{P_{j}}$

where the expectation is taken over the data bits, phase offsets and delays, each of which forms a set of independent random variables which are also mutually independent. Note that cross-terms in the squared expression are the product of independent, zero mean random variables. Averaging over the initial phase offsets $\theta_{i k}$, we can write

$$
\operatorname{var}\left[I_{j l}\right]=\sum_{\substack{i=1 \\(i k j l)}}^{p} \sum_{k=1}^{K_{i}} \frac{1}{2 T_{j}^{2}} \frac{P_{i}}{P_{j}} E_{b_{i k}, \tau_{i k}}\left[J_{i k}^{2}\right]
$$

and define the cross-correlation

$$
J_{i k}={ }_{\boldsymbol{d}^{T_{j}}} b_{i k} \boldsymbol{D}_{\tau_{i k}} \boldsymbol{g}_{k} \boldsymbol{D}_{\tau_{i k}} \boldsymbol{g}_{j l} \mathbf{b d}
$$

We further define the partial cross-correlation function as

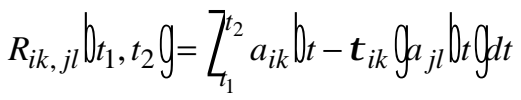

The integral of (10) is defined over $\left[0, T_{j} \mathbf{i}\right.$ but the delays are defined over $\left[0, T_{i} \mathbf{l}\right.$, this means that to solve equation (9) it is necessary to divide further calculations into three distinct cases: $T_{j}=T_{i}, T_{j}<T_{i}$ and $T_{j}>T_{i}$

\subsubsection{Case $T_{j}=T_{i}$}

This is the classic single rate case $[1,1,2]$, all interfering signals' sequence lengths being equal to the desired signal's sequence length (i.e., $N_{j}=N_{i}$ ). $J_{i k}$ can be written as [1]

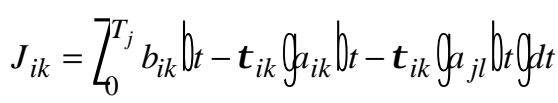

where $b_{i k}^{(-1)}$ and $b_{i k}^{(0)}$ are interfering bits overlapping the desired signal's bit $b_{j l}^{(0)}$. Define $C_{x y}()$, the aperiodic crosscorrelation function, by

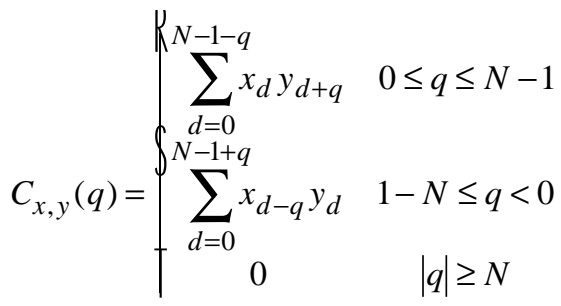

where $N$ is the length of the code sequences and $x_{d}$ is the $d^{\text {th }}$ chip of sequence $x$. It has been shown $[1,1,2]$ that

$$
E_{b_{i k}, \tau_{i k}}\left[J_{i k}^{2}\right]=\frac{T_{c}^{3}}{3 T_{i}} \cdot r_{i k}
$$

The interference parameter $r_{i k}$ is the link between the discrete (chip synchronous) aperiodic cross-correlation function that we can easily evaluate numerically and the partial cross- 
correlation function that will enable us to evaluate the BER performance of the asynchronous network.

$$
\begin{aligned}
r_{i k} & =\sum_{q=0}^{N_{i}-1}\left\{C_{i k, j l}\left(q-N_{i}\right)^{2}+C_{i k, j l}\left(q+1-N_{i}\right)^{2}\right. \\
& +C_{i k, j l}\left(q-N_{i}\right) C_{i k, j l}\left(q+1-N_{i}\right) \\
& \left.+C_{i k, j l}(q) C_{i k, j l}(q+1)+C_{i k, j l}(q)^{2}+C_{i k, j l}(q+1)^{2}\right\}
\end{aligned}
$$

\subsubsection{Case $T_{j}>T_{i}$}

The first multi-rate case we examine has the desired user's bit being longer than the interferer's bit. We use the definition of the ratio of chip rates $L$ to write (10) as

$$
\begin{aligned}
J_{i k}= & \sum_{m=0}^{L-2} b_{i k}^{(m)} R_{i k, j l} \boldsymbol{\epsilon}_{i k}+m T_{i}, \tau_{i k}+\mathbf{b}_{+1} \mathbf{g h} \\
& +b_{i k}^{(-1)} R_{i k, j l} \mathbf{Q}_{\tau_{i k}} \mathbf{b}_{i k}^{(L)} R_{i k, j l} \mathbf{d}_{k}+L T_{i}, T_{j} \mathbf{j} \\
& +b_{i k}^{(L-1)} R_{i k, j l} \boldsymbol{\epsilon}_{i k}+\mathbf{b}_{-1} \mathbf{g}, \tau_{i k}+L T_{i} \mathbf{h}
\end{aligned}
$$

where Erreur! Source du renvoi introuvable. illustrates the many overlapping bits contributed by each interfering user to calculate $J_{i k}$. It is necessary to average $J_{i k}$ over these bits and their delays. When squaring $J_{i k}$, the cross-terms are the product of independent, zero mean random variables, so the variance of $J_{i k}$ is

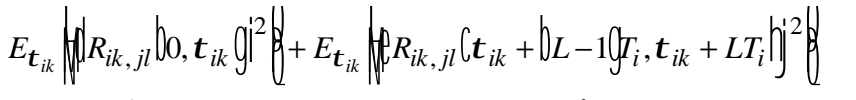

$$
\begin{aligned}
& +\sum_{m=0}^{L-2} E_{\tau_{i k}} \mathbf{b}_{k, k l}+m T_{i}, \tau_{i k}+(m+1) T_{i} \mathbf{Q} \\
& +E_{\tau_{i k}} \operatorname{dat}_{k, j l} \mathbf{d}_{k}+L T_{i}, T_{j} i^{2} \boldsymbol{P}
\end{aligned}
$$

We divide the integral into a sum of integrals over the chip intervals. We can express the partial cross-correlations as

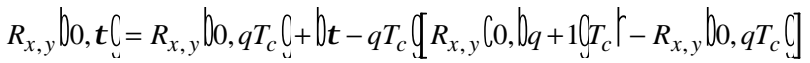

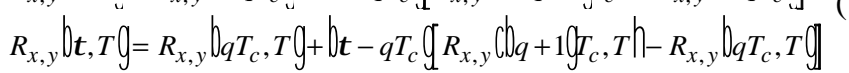

as in the single user case [1]. We define the aperiodic crosscorrelation function for $T_{j}>T_{i}$ as

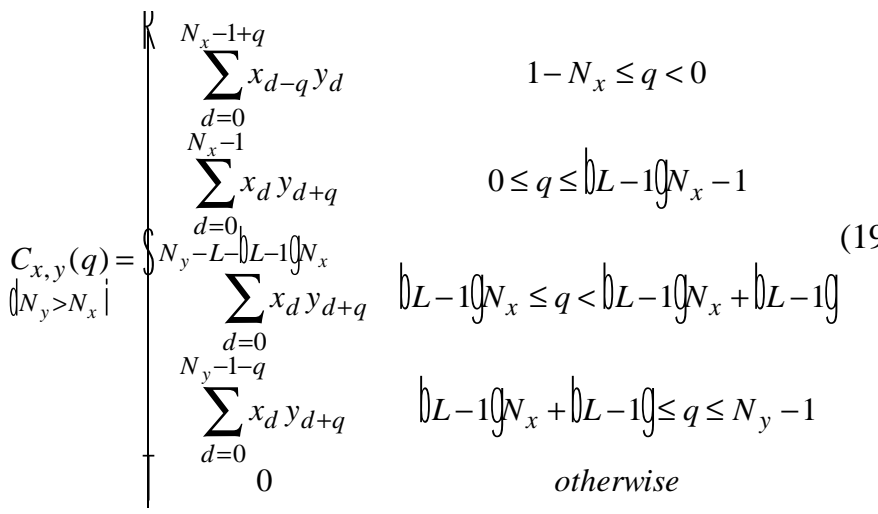

We note that for $N_{x}=N_{y}$ the equation reduces the single user case. With this definition the equations for the partial crosscorrelations become

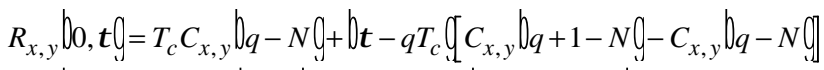

$$
\begin{aligned}
& R_{x, y} b_{T} \dot{\mathbf{g}} T_{c} C_{x, y} \operatorname{bg} \dot{b}_{-q T_{c}} \mathbf{g}_{x, y} \mathbf{b}_{+1} \mathbf{g} c_{x, y} \dot{\mathbf{b g}}
\end{aligned}
$$

While these two expressions are identical to those for the single user case, we note that the aperiodic cross-correlation function $C_{x y}$ differs greatly from the single user case. After substituting (20) into (17) and extensive algebraic manipulation (verified via symbolic mathematics software) we can write

$$
E_{b_{i k}, \tau_{i k}}\left[J_{i k}^{2}\right]=\frac{T_{c}^{3}}{3 T_{i}} \cdot r_{i k}
$$

where the interference parameter for this case is as follows.

$$
\begin{gathered}
r_{i k}=\sum_{q=0}^{N_{i}-1}\left\{C_{i k, j l}\left(q-N_{i}\right)^{2}+C_{i k, j l}\left(q+1-N_{i}\right)^{2}\right. \\
\left.+C_{i k, j l}\left(q-N_{i}\right) C_{i k, j l}\left(q+1-N_{i}\right)\right\} \\
+\sum_{m=0}^{L}\left\{C_{i k, j l}\left(q+m N_{i}\right)^{2}+C_{i k, j l}\left(q+1+m N_{i}\right)^{2}\right. \\
\left.+C_{i k, j l}\left(q+m N_{i}\right) C_{i k, j l}\left(q+1+m N_{i}\right)\right\}
\end{gathered}
$$

\subsubsection{Case $T_{j}<T_{i}$}

Using the same analytical approach it can be shown that

$$
E_{b_{i k}, \tau_{i k}}\left[J_{i k}^{2}\right]=\frac{T_{c}^{3}}{3 T_{i}} \cdot r_{i k}
$$

where the interference parameter is

$$
\begin{gathered}
r_{i k}=\sum_{q=N_{j}}^{N_{i}-1}\left\{C_{i k, j l}\left(q-N_{i}\right)^{2}+C_{i k, j l}\left(q+1-N_{i}\right)^{2}\right. \\
\left.\quad+C_{i k, j l}\left(q-N_{i}\right) C_{i k, j l}\left(q+1-N_{i}\right)\right\} \\
+\sum_{q=0}^{N_{j}-1}\left\{C_{i k, j l}\left(q-N_{i}\right)^{2}+C_{i k, j l}\left(q+1-N_{i}\right)^{2}\right. \\
+C_{i k, j l}\left(q-N_{i}\right) C_{i k, j l}\left(q+1-N_{i}\right)+C_{i k, j l}(q)^{2} \\
\left.+C_{i k, j l}(q+1)^{2}+C_{i k, j l}(q) C_{i k, j l}(q+1)\right\}
\end{gathered}
$$

and the aperiodic cross-correlation function is

$$
\mathbf{d}_{y}<N_{x} \mathbf{i}(q)=\left\{\begin{array}{cc}
\sum_{d=0}^{N_{y}-1-q} x_{d} y_{d+q} & 1 \leq q \leq N_{y}-1 \\
N_{x}-1+q & \\
\sum_{d=0}^{N_{y}-1} x_{d-q} y_{d} & 1-N_{x} \leq q \leq N_{y}-N_{x}-1 \\
0 & N_{y}-N_{x} \leq q \leq 0 \\
0 & \text { otherwise }
\end{array}\right.
$$




\subsection{Signal to Noise + Interference Ratio (SNIR)}

We are now in a position to take the variance of the MAI and write an expression for the signal-to-noise-plus-interference ratio (SNIR). In all cases of relative sequence length $\left(T_{i}=T_{j}\right.$, $T_{i}<T_{j}, T_{i}>T_{j}$ ) we found

$$
E_{b_{i k}, \tau_{i k}}\left[J_{i k}^{2}\right]=\frac{T_{c}^{3}}{3 T_{i}} \cdot r_{i k}
$$

Therefore we can write

$$
\operatorname{var}[\mathrm{MAI}]=\operatorname{var}\left[I_{j l}\right]=\sum_{\substack{i=1 \\(i k \neq j l)}}^{p} \sum_{k=1}^{K_{i}} \frac{1}{6 N_{i} N_{j}^{2}} \frac{T_{j}}{T_{i}} r_{i k}
$$

Equation (24) gives us an expression of the MAI in terms of the relative transmission rates and the interference parameter $r_{i k}$ that is only a function of the code sequences (of various lengths). The SNIR is given by

$$
\text { SNIR }_{\text {multirate }}=\frac{1}{\sum_{\substack{i=1 \\(i k \neq j l)}}^{p} \sum_{k=1}^{K_{i}} \frac{1}{6 N_{i} N_{j}^{2}} \frac{T_{j}}{T_{i}} r_{i k}+S N R}
$$

where $S N R$ is the single-user signal-to-thermal-noise ratio for the network. The probability of error function for BPSK, using the Gaussian assumption for the MAI, is

$$
P_{e}=0.5 \text { erfc }
$$

With equation (26) we have an expression for the probability of error $(B E R)$ as a function of the interference parameter for specific code sequences.

\section{Calculation Results}

As seen in (25), the SNIR (and hence the BER) depends on the set of interference parameters $r_{i k}$ for all the active users. Since the set of active users is time-varying and we wish to know overall performance of the system, we must do some type of averaging over the interference parameter. Previous work for both single-rate [1] and multi-rate [3] CDMA has accomplished this averaging by modeling the spreading codes as truly random. The variance of the MAI is then determined by further averaging over the random chip sequences. Since the interference parameter appears linearly in the MAI variance, this amounts to calculating the mean of (15), (21) or (22) under the random code assumption. We calculate this to be

$$
E_{\text {random codes }} r_{i k}= \begin{cases}2 N_{i}^{2} & T_{j}=T_{i} \\ L N_{i}^{2} & T_{j}>T \\ 2 N_{j} N_{i} & T_{j}<T\end{cases}
$$

corroborating the results in [1] and [3]. The mean no longer depends on the subscript $k$, as all codes have the same mean.

We calculate the value of all possible interference parame- ters $r_{i k}$ for various code sets and examine the histograms of these calculations to 1) gauge the accuracy of the random code assumption, and 2) determine the probability density function (pdf) of the interference parameter. Knowledge of the pdf of the interference parameter allows us to go beyond simply using the average value of the SNIR to calculate the BER. With the pdf we can also generate confidence intervals for the BER calculations.

Table 1 Sample mean and variance of the average interference parameter $\left(r_{i k}\right)$ for various sequences

\begin{tabular}{|l|l|l|l|c|c|c|}
\hline Case & \multicolumn{2}{|c|}{ Desired user } & \multicolumn{2}{c|}{ Interferer } & \multicolumn{2}{c|}{$r_{i k}$} \\
& \multicolumn{1}{|c|}{ Type } & $N_{j}$ & \multicolumn{1}{c|}{ Type } & $N_{i}$ & \multicolumn{1}{c|}{ mean } & var \\
\hline 1 & Gold & 127 & Gold & 127 & 0.9921 & 0.1079 \\
\hline 2 & Gold & 511 & Gold & 127 & 1.0032 & 0.0707 \\
\hline 3 & Gold & 511 & Kasami & 255 & 0.9966 & 0.0684 \\
\hline 4 & Gold & 127 & Gold & 511 & 1.0000 & 0.0807 \\
\hline 5 & Gold & 127 & Kasami & 255 & 0.9947 & 0.0966 \\
\hline
\end{tabular}

In Table 1 we present the sample mean and variance of the interference parameter for several code lengths and code types. Case 2 is a multi-rate $\left(T_{j}>T_{i}\right)$ system with Gold codes of length 127 and 511. Case 3 is a multi-rate $\left(T_{j}>T_{i}\right)$ system with Gold codes of length 511 and Kasami codes of length 255. Case 4 has Gold codes of length 127 and 511, as in case 2, however the desired user now has code length 127 , i.e., $T_{j}<T_{i}$. Case 5 uses the same codes as case 3 , however the desired user's code is length 127, i.e., $T_{j}<T_{i}$. In Figure 1 we plot the histograms of the interference parameter for a single-rate $\left(T_{j}=T_{i}\right)$ system (case 1) of Gold codes of length 127.

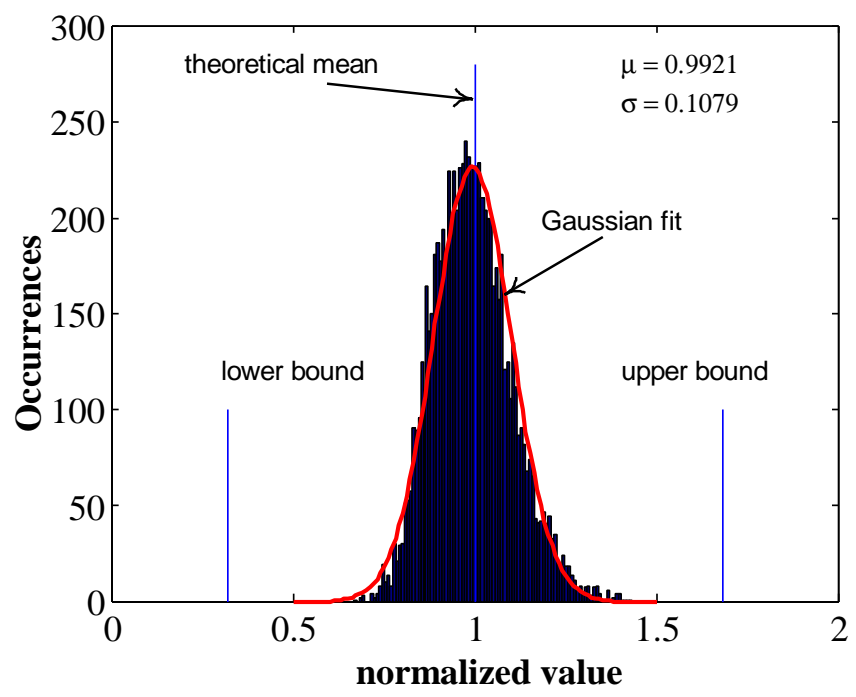

Figure 1 Average interference parameter $\left(r_{i k}\right)$ for Gold sequences; $N_{j}=N_{i}=127$

In Figure 1 as well as in Table 1, we have normalized the interference parameter to its mean under the random code assumption. That is, a sample mean of $\mu=1.0$ corresponds to perfect coincidence with the theoretical mean. The sample 
mean was seen to vary between .9921 and 1.0032 . The histogram in Figure 1 also shows that previous lower and upper bounds are quite wide compared with the lower/upper limits of the distributions. We observed that the pdf of the interference parameter closely resembled that of the Gaussian distribution. In Figure 1 we include a Gaussian pdf using the sample mean and variance.

Having determined by calculation the pdf of the interference parameter, we next calculate the confidence intervals for the BER determined using the random code assumption, i.e., using the mean of the interference parameter. Recall that for a length 127 code and a code set of 127 codes, there are $126 \times 127$ interference parameters. For example, for twelve active users only twelve of these interference parameters would contribute to the MAI variance, and their values could vary widely from the mean. The pdf shows how large the deviation is likely to be.

We now assume that our interference parameters $r_{i k}$ are indeed well modeled as a set of independent Gaussian random variables. That is, for a given value of $i$, for a randomly chosen set of active users, their interference parameters are independent and identically distributed. We define

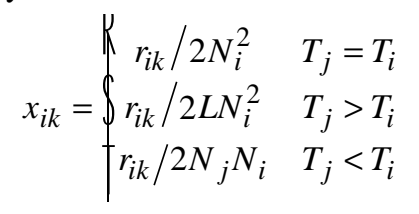

which will nominally have unity mean. The observed means and variances of these Gaussian random variables are determined by the previous exhaustive calculations and are indicated in Table 1 for the particular code sequences studied.

$$
\text { Using equation (27) we can define }
$$

We assume the parameters $x_{i k}$ are uncorrelated, so that their moments are easily calculated. Given equation (26) we can plot confidence intervals for the probability of error using the distribution of $X$,

$$
P_{e}=0.5 \text { erfc } \frac{1}{2}
$$

where $X$ is replaced by its mean, then by its mean plus one standard deviation, then by its mean minus one standard deviation. This gives a confidence interval of $68 \%$. For $95 \%$ confidence we add/subtract twice the standard deviation.

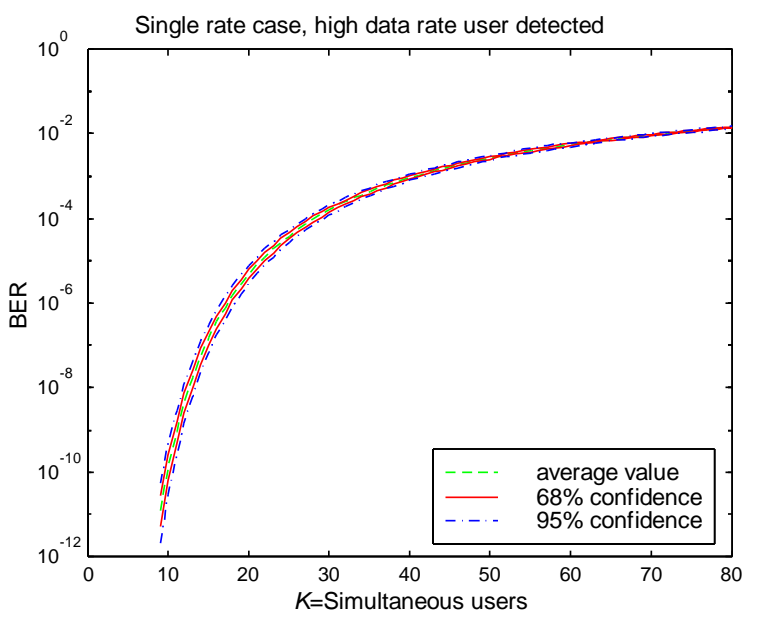

Figure 2 Single rate system, high data rate user detected, case 1

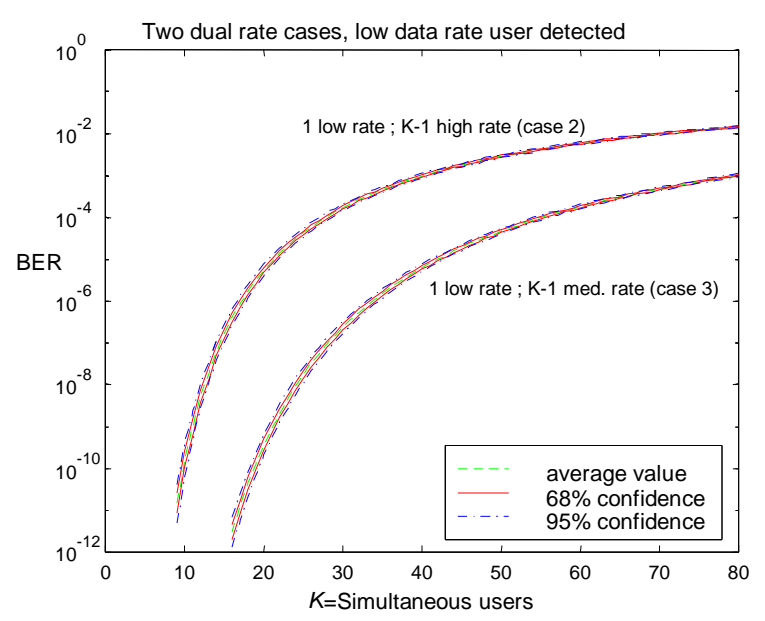

Figure 3 Dual rate systems, low data rate user detected, cases $2 \& 3$, one low data rate user, $K-1$ other rate users

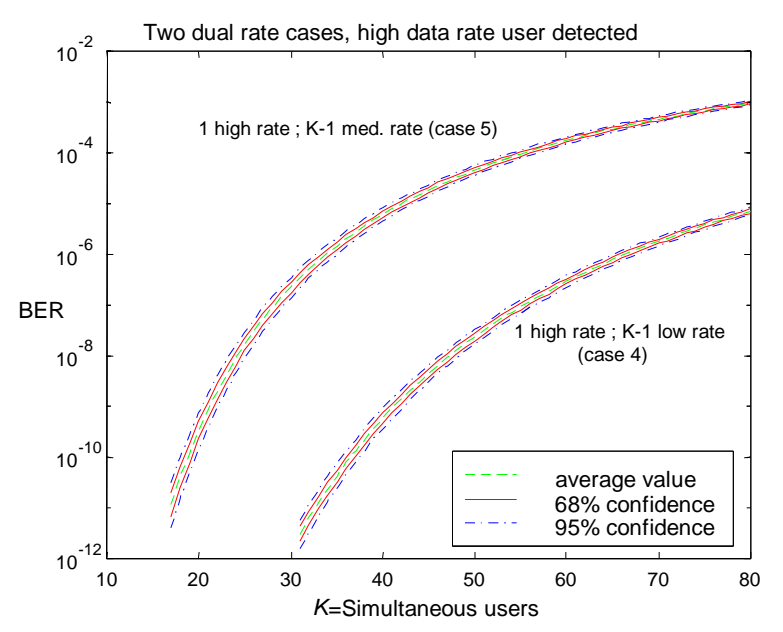

Figure 4 Dual rate systems, high data rate user detected, cases $4 \& 5$, one high data rate user, K-1 other rate users 
Note that under the random code assumption, the mean of the interference parameter is the same for all codes, therefore the BER is a function of the number of active users, $K$. For our observation of a Gaussian fit to the interference parameter, the BER depends on the number of users at each data rate, $K_{i}$. We will plot cases for systems with two and three data rates, using the calculation for the Gaussian pdf presented in Table 1.

For Figures 2-7 we fix the chip rate and the single-user SNR (i.e., the signal to thermal noise ratio) and plot the BER versus the number of simultaneous users $K$, with all data taken from Table 1. A fairly strong SNR of $28 \mathrm{~dB}$ was used to stress conditions under which the MAI is the most important noise source.

Using the Gaussian fit of the interference parameter pdf, we calculate the bounds on the parameter for $95 \%$ confidence and $68 \%$ confidence, and plot the BER for the average value, and the extreme values for these confidence intervals. In Figure 2 we see a single rate system, and the confidence intervals are very tight, confirming the mean is a good estimator of the interference parameter for single rate systems. In Figure 3 and Figure 4 we present dual data rate systems, but with the desired user the only user at one of the two data rates. Here too the confidence intervals are reasonably tight, although we begin to see some divergence in Figure 5. In Figure 6 we take one of the dual rate systems and let the occupation of active users at each data rate vary. As we let the number of high rate users increase from the single desired user to half the active users, we see that the confidence interval can become significantly wide. For instance, for a BER of $10^{-10}$ the number of users can vary from 28 to 33. In Figure 6 and Figure 7 we see similar behavior in a three-rate system with various occupancies at each rate.

\section{Conclusions}

Our simulations demonstrate a near Gaussian distribution of the values of $r_{i k}$ when all combinations of sequences and delays are considered. The deviation from the mean value can be quantified by the use of a confidence interval in the evaluation of the BER of a multi-rate CDMA system. The results of our calculations confirm that in single rate systems the random sequence assumption is a good estimator of the mean value of $r_{i k}$. On the other hand, some discrepancies can be seen in the multi-user case, i.e., significant variation from the mean.

\section{References}

1. M. B. Pursley, "Performance Evaluation for Phase-coded Spread Spectrum Multiple Access Communication-Part I: System Analysis," IEEE Transactions on Communications, vol. 25, no. 8, pp. 795-799, August 1977.

2. M. B. Pursley and D. V. Sarwate, "Performance Evaluation for Phase-coded Spread Spectrum Multiple Access Communication-Part II: Code Sequence Analysis," IEEE Transactions on Communications, vol. 25, no. 8, pp. 800-803, August 1977.

3. T. Ottoson and A. Svensson, "Multi-rate Schemes in DS/CDMA Systems," Proceedings IEEE VTC'95, Chicago, pp. 1006-1010, July 1995.

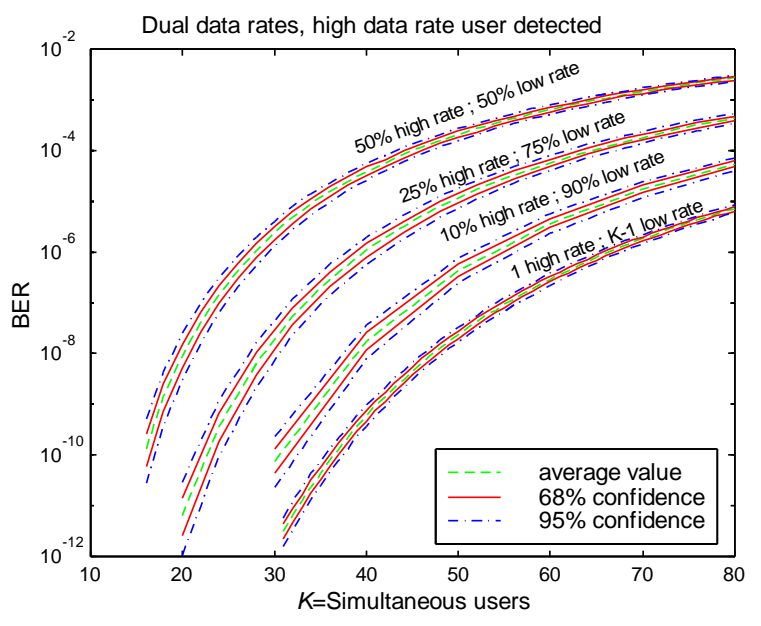

Figure 5 Dual rate system, high data rate user detected, case 4 , various rate occupancies

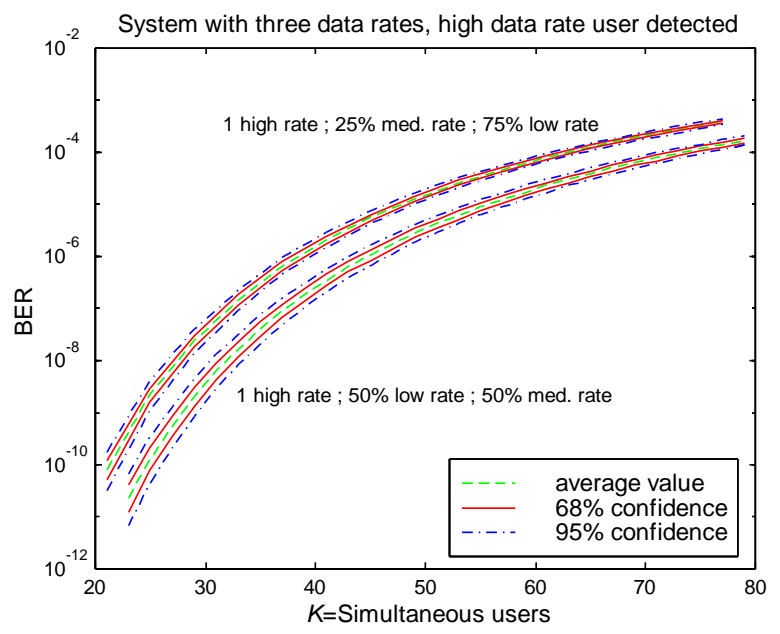

Figure 6 System with three data rates, high data rate user detected, case 4 , one high rate user, various rate occupancies 


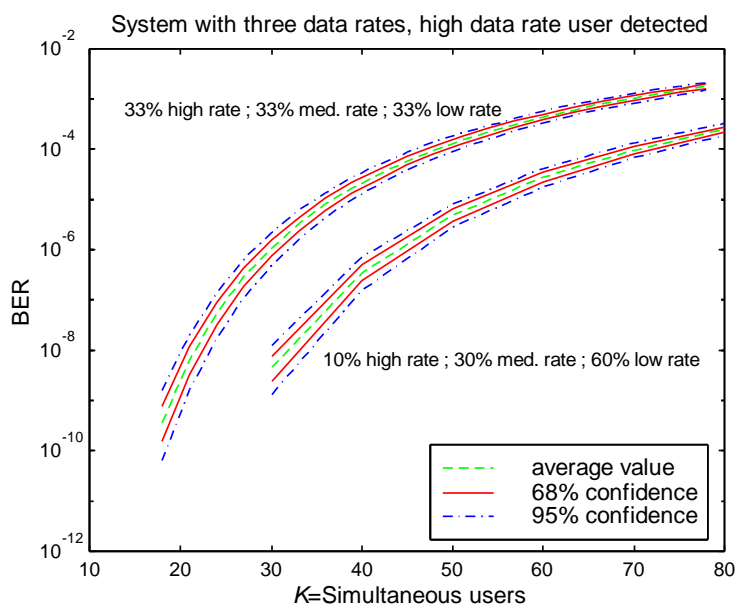

7. Figure 7 System with three data rates, high data rate user detected, case 4 , various rate occupancies 